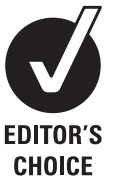

Health Economist, Oxford Outcomes Ltd, Oxford, UK ${ }^{2}$ German Heart Center, Munich, Germany

${ }^{3}$ Department of Cardiac Surgery, Royal Brompton Hospital, London, UK

${ }^{4}$ Medtronic International Trading Sàrl, Tolochenaz, Switzerland ${ }^{5}$ Centre for Health Economics, University of York, York, UK

\section{Correspondence to} Maureen Watt, Oxford Outcomes Ltd, West Way, Botley, Oxford OX2 0JJ, UK; maureen.watt@ oxfordoutcomes.com

Accepted 23 September 2011 Published Online First 10 November 2011

\title{
Cost-effectiveness of transcatheter aortic valve replacement in patients ineligible for conventional aortic valve replacement
}

\author{
Maureen Watt, ${ }^{1}$ Stuart Mealing, ${ }_{1}$ James Eaton, ${ }^{1}$ Nicolo Piazza, ${ }^{2}$ Neil Moat ${ }^{3}$ \\ Pascale Brasseur, ${ }^{4}$ Stephen Palmer, ${ }^{5}$ Rachele Busca, ${ }^{4}$ Mark Sculpher ${ }^{1,5}$
}

\begin{abstract}
Objective To assess the cost-effectiveness of transcatheter aortic valve implantation (TAVI) compared with medical management in patients with severe aortic stenosis who are ineligible for conventional aortic valve replacement (SAVR) from the perspective of the UK

National Health Service.

Design Probabilistic decision analytical model.

Methods A decision analytical model was developed to assess the costs and benefits associated with both interventions over a 10-year time horizon. A literature review was performed to identify relevant clinical evidence. Health-related quality of life and mortality were included using data from the PARTNER clinical trial (cohort B). Unit costs were taken from national databases. Costs and benefits were discounted at 3.5\% per year, and extensive sensitivity analyses (probabilistic and deterministic) were performed to explore the impact of uncertainty on the cost-effectiveness estimates.
\end{abstract}

Main outcome measure Incremental costeffectiveness ratio (ICER) with benefits expressed as quality-adjusted life years (OALYS).

Results The base case ICER was approximately £16 100 per QALY gained. At a cost-effectiveness threshold of $£ 20000$ per QALY gained, the probability that TAVI was cost-effective compared with medical management was 1.00. The results were robust to changes in key clinical parameters as well as choice of baseline survival data. The observed PARTNER survival data only have to be extrapolated for 2 years to generate an ICER below $£ 30000$ per QALY gained, which is the upper value of the threshold range used by the National Institute for Health and Clinical Excellence in the UK.

Conclusions TAVI is highly likely to be a cost-effective treatment for patients with severe aortic stenosis who are currently ineligible for SAVR.

\section{INTRODUCTION}

Aortic stenosis is a common condition affecting approximately $3 \%$ of individuals aged $>75$ years. ${ }^{1}$ After the onset of symptoms or left ventricular dysfunction, prognosis is poor with reported 2-year mortality estimates approaching $50 \%{ }^{2-4}$

The current gold standard of treatment for patients with severe symptomatic aortic stenosis is surgical aortic valve replacement (SAVR). ${ }^{5}$ This procedure has been shown to reduce symptoms and to improve survival. ${ }^{6}$ Despite the existence of this effective treatment option, a significant number of patients are deemed to be unsuitable for SAVR (for a multitude of reasons including advanced age and the presence of multiple comorbidities). Although balloon aortic valvuloplasty (BAV) was once considered an alternative treatment option for patients with inoperable aortic stenosis, restenosis and recurrent symptoms occurred in $80 \%$ of patients by $6-12$ months follow-up.

In 2002 Cribier et al performed the first transcatheter aortic valve implantation (TAVI) for patients with inoperable aortic stenosis. ${ }^{5}$ Evidence relating to the efficacy of TAVI in this patient group has been emerging over time but, until recently, has been largely limited to single-arm studies, retrospective registry analyses and conference proceedings. ${ }^{7-13}$

The results of the Placement of Aortic Transcatheter Valves (PARTNER US, cohort B) randomised clinical trial have recently been published by Leon et $a l^{14}$ and substantiated the hypothesised positive treatment effect seen in the retrospective registry analyses. ${ }^{8}$ Individuals were randomised to receive either TAVI or standard therapy (ie, pharmacological, but often including BAV). After 12 months, TAVI was shown to have a significantly beneficial impact on mortality, repeat hospitalisations and functional status of the patients $(p<0.001$ for all three endpoints).

TAVI can, however, be viewed as an expensive procedure to perform in elderly patients with multiple comorbidities. Despite promising clinical results, in countries such as the UK where healthcare is collectively funded, it is one of many health technologies competing for funding from a limited healthcare budget. We therefore built a cost-effectiveness model from a UK perspective to compare the costs and benefits of medical management versus TAVI in patients with inoperable aortic stenosis.

\section{METHODS}

\section{Model description}

The analysis was based around two interlinked Markov models designed to represent the shortand long-term periods of time following surgery. Briefly, in this approach the underlying disease and/ or patient pathway is represented by a series of health states between which patients move in discrete intervals of time (the cycle length) for a number of cycles (the time horizon). The Markov models used in the analysis presented in this paper 
were implemented in Microsoft Excel (Microsoft Corporation, Redmond, Washington, USA). The model structure (figure 1) and its underlying assumptions were developed in discussion with an expert advisory group comprising clinical and economic advisors with experience in clinical trials design and health economic modelling.

In the 'short-term' model we used a time horizon of 30 days and a cycle length of 1 day. Health states were based around location of care (intensive care (ICU), non-ICU and nonhospital) as well as reoperations and post-hospital rehabilitation (community and managed). Death was possible from all health states in all cycles. All patients in the TAVI arm were assumed to incur the cost of the initial implant procedure. $83 \%$ of individuals in the medical management arm were assumed to receive a BAV procedure and all incurred the costs of pharmacological treatment. $^{14}$

Contingent on being alive at 30 days, all patients moved to the 'long-term' model containing health states corresponding to home care, reoperation and death. An alternative cycle length of 1 month was used in combination with a 10-year time horizon.

Patients in each treatment arm accrued costs and benefits (quality-adjusted life years, OALYs) as they passed through these states. Discount rates of $3.5 \%$ per annum were applied to the total costs and OALYs accrued. The results are presented as an incremental cost-effectiveness ratio (ICER) expressed as the ratio of incremental costs to incremental benefits. A UK National Health Service (NHS) perspective was used and thus only direct healthcare expenditure was included.

The methods used to estimate key parameters are summarised below. All values were identified by a concomitant literature review.

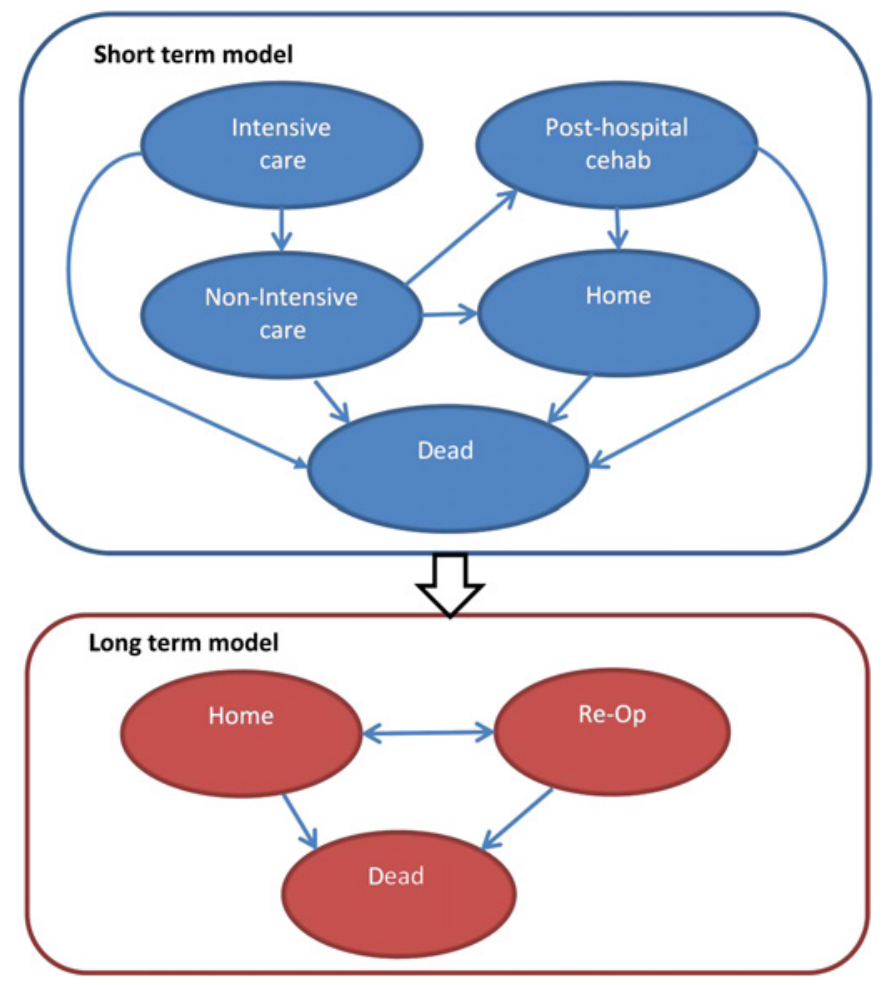

Figure 1 Model schematic. Note that all individuals move into the relevant health state in the long-term model at the end of the short-term model. In addition, all individuals in the medical management arm begin in the 'HOME' state and cannot enter the 'Re- $0 p^{\prime}$ ' state when in the longterm model.

\section{Description of model inputs}

\section{Estimating mortality}

In order to generate lifetime cost and benefit estimates, the extrapolation of survival data observed in clinical trials is inevitable. The most relevant and up to date information is available from the PARTNER US clinical trial, ${ }^{14}$ with information on death from any cause being available for up to 24 months in each treatment arm.

Conventionally, this extrapolation process is undertaken through the use of a mathematical function to approximate the baseline or comparator event rate, and a hazard ratio (HR) which is applied to the fitted function in order to approximate the survival rate in the treatment arm. These functions are then used to generate survival estimates for all time points in the model. Underpinning this approach, however, is the assumption that, at any given time point, the rate of death in the treatment arm is proportional to that in the comparator arm.

The published Kaplan-Meier survival curves were electronically extracted and, on the basis of an analysis of the hazard functions, the assumption of proportional hazards was shown not to hold. Independent parametric survival functions were therefore fitted to each published curve. Monthly or daily transition probabilities were derived as required from the fitted functions using standard formulae for each cohort. For patients receiving TAVI, the daily probabilities were based on intraoperative mortality, 30-day survival in the medical management arm and the short-term treatment effect.

Of the patients receiving TAVI in the PARTNER US study, $1.1 \%$ died intraoperatively and this value was used directly in the model for the appropriate parameter.

\section{Estimating other clinical events}

Where possible, the values used for key treatment-related adverse events (stroke, bleeding, vascular events, renal failure and aortic regurgitation), the proportion requiring additional pacing and the expected length of stay in the ICU and non-ICU were derived from the study by Leon et al. ${ }^{14}$ In the absence of any reported information, we used values estimated by the clinical steering group. Transition between ICU, non-ICU and home were derived from the mean length of stay estimates and assumptions of uniform rate of movement. A minimum length of hospitalisation was also included. The values used in the model are presented in table 1 . The reliance on early stage TAVI data (as reported in Leon et al) may mean that the values used for a number of clinical parameters are higher than those observed in routine UK clinical practice. The resulting costeffectiveness results will therefore be biased against TAVI and hence conservative.

The monthly probability of heart failure-related rehospitalisation in the medical management arm, as well as the TAVI treatment effect, were calibrated to ensure the 1 year rates were identical to those reported by Leon et al. ${ }^{14}$

As with intraoperative mortality, a literature review was performed to identify additional studies that could be used to inform these transition probabilities. Pooled estimates covering different devices and procedures were used in sensitivity analyses (table 1).

\section{Costs and health-related quality of life weights}

Drug costs were taken from the most recent edition of the British National Formulary ${ }^{18}$ and, where possible, all unit costs were taken from other publically available national databases. ${ }^{16}{ }^{17}$ Reported NHS unit costs for excess bed days were used in combination with the adverse event rates to generate treatment-specific costs for 
Table 1 Key parameters used in model

\begin{tabular}{|c|c|c|c|c|}
\hline \multirow[b]{2}{*}{ Parameter } & \multicolumn{2}{|c|}{ Base case analysis } & \multicolumn{2}{|c|}{ Sensitivity analysis } \\
\hline & Value & Source & Value & Source \\
\hline \multicolumn{5}{|l|}{ Length of stay } \\
\hline Minimum hospital stay & 5 days & Expert opinion & 5 days & Expert opinion \\
\hline Mean ICU LOS & 2 days & Expert opinion & 2 days & Literature review \\
\hline Mean GW LOS* & 7 days & Expert opinion & 7 days & Literature review \\
\hline \multicolumn{5}{|l|}{ Event probabilities } \\
\hline Conversion to SAVR & $0.0 \%$ & 14 & $1.78 \%$ & Literature review \\
\hline Intraoperative death & $1.1 \%$ & 14 & $1.45 \%$ & Literature review \\
\hline Reoperation (short-term) & $1.7 \%$ & 14 & $2.36 \%$ & Literature review \\
\hline Stroke $†$ & $6.70 \%$ & 14 & $2.88 \%$ & Literature review \\
\hline Bleeding $†$ & $16.8 \%$ & 14 & $10.06 \%$ & Literature review \\
\hline Vascular† & $30.70 \%$ & 14 & $7.52 \%$ & Literature review \\
\hline Aortic regurgitation $\dagger$ & $11.80 \%$ & 14 & $23.39 \%$ & Literature review \\
\hline Renal failure $\dagger$ & $1.1 \%$ & 14 & $8.87 \%$ & Literature review \\
\hline Pacemaker implant $† \neq$ & $3.4 \%$ & 14 & $7.70 \%$ & Literature review \\
\hline \multicolumn{5}{|l|}{ Unit costs } \\
\hline Procedure cost (per hour) & & & $£ 524.40$ & 15 \\
\hline Day in ICU & & & $£ 1.149$ & 16 \\
\hline Day in non-ICU & & & $£ 136$ & 16 \\
\hline Pacemaker implantation & & & $£ 3119-£ 6371 \S$ & 16 \\
\hline Community-based rehabilitation & & & $£ 1575$ & 17 \\
\hline Managed rehabilitation & & & $£ 4081$ & 17 \\
\hline
\end{tabular}

a day in the non-ICU health state. The total cost of a TAVI operation included all procedure and device costs, with procedure times and costs taken from a recent costing study. ${ }^{15}$ Where necessary, values were inflated to 2010 levels using an appropriate inflation index. ${ }^{17}$

Health-related quality of life (HROoL) was incorporated into the model as utility values expressed on a scale of $0-1$ (where 1 is equivalent to perfect health and 0 is equivalent to dead). HRQoL information was not available from the PARTNER US study at the time of model construction so an alternative approach was used. Baseline age- and gender-adjusted EQ-5D (EuroQol) values for the general UK population were derived by fitting using a mathematical function. ${ }^{19}$ The impact of the underlying disease was incorporated into the model as an HRQoL decrement $(-0.197)$ derived from information on baseline New York Heart Association (NYHA) class mix and published EQ-5D preference weights. ${ }^{20}$ Information from PARTNER US on the NYHA class mix at given time points was used to generate time-dependent utility decrements (and hence include the effect of treatment into the model). Decrements for patients in the ICU and non-ICU health states were derived from the literature. ${ }^{21}$

All decrements were applied to the age- and gender-adjusted population EQ-5D norms during each model cycle to generate the time-dependent utility values in each arm.

As a sensitivity analysis, information from a relevant clinical trial $^{22}$ was used to inform a mathematical function assessing the time-dependent change of utility in patients receiving TAVI. The EQ-5D population norms were applied as a ceiling to the derived values from this function. Alternative functions and decrements were explored in sensitivity analyses.

For an additional sensitivity analysis, the information reported by Leon et $a l^{14}$ on NYHA class at four time points (baseline, 30 days, 6 months, 1 year) for both treatment options was used in combination with the published NYHA values. $^{20}$

\section{Device longevity}

Pacemaker lifetimes were assumed to be the same as in a previous UK-based health technology assessment. ${ }^{20}$ The lifetime of a TAVI was derived from long-term information on the failure of standard bioprosthesis devices. ${ }^{23}$ A Weibull function was fitted to the reported data and we made the arbitrary assumption that, in any given month, the probability of TAVI failure would be twice the derived value. All of these values were altered in sensitivity analyses.

\section{Sensitivity analyses}

We conducted a probabilistic sensitivity analysis whereby individual sets of parameter values were drawn from appropriate statistical distributions, with results generated for 1000 simulation runs. Cost-effectiveness acceptability curves were generated to represent decision uncertainty-the probability that TAVI is cost-effective conditional on a range of possible costeffectiveness thresholds. ${ }^{24}$ In addition, a range of deterministic analyses was performed to explore the impact of alternative parameter values and modelling assumptions on model results.

In particular, an assessment of the impact of alternative choices of different clinical trials to inform baseline (ie, medical management) mortality on the cost-effectiveness of TAVI was also performed. The literature review identified three possible alternative sources. $^{8} 2526$ Comparison of the 2-year mortality estimates from these three trials (either directly or via the fitting of parametric survival functions) with the value from Leon et al ${ }^{14}$ provides 'scaling factors' (multipliers) which can be used to perform such an analysis. 


\section{RESULTS}

The costs and benefits generated when the model was run probabilistically are presented in table 2 . In comparison to medical management, individuals in the TAVI arm incurred an additional 10 -year cost per patient of $£ 25200$. The majority of TAVI-related costs correspond to the initial implant operation (£19000) or perioperative ICU care (£2500). However, the use of TAVI conferred savings in terms of BAV procedures avoided (-£2400). TAVI also conferred an additional 1.56 OALYs over 10 years. Thus, the base case ICER is $£ 16200$ per QALY gained.

The cost-effectiveness acceptability curves for medical management and TAVI are presented in figure 2A. Assuming a cost-effectiveness threshold of $£ 20000$ per OALY gained, the probability that TAVI is a cost-effective intervention in the target patient group is 1.00 .

\section{Alternative assumptions and scenarios}

Use of pooled estimates for key model parameters

When the survival estimates are unchanged but the pooled input parameters rather than those observed in PARTNER are used,

Table 2 Key economic outputs (base case and sensitivity analyses)

\begin{tabular}{|c|c|c|c|c|}
\hline Arm & \multicolumn{2}{|c|}{ Costs* } & OALYs & $\begin{array}{l}\text { ICER } \\
\text { ( }(\text { /OALY gained) }\end{array}$ \\
\hline \multicolumn{5}{|l|}{ Base case $\dagger$} \\
\hline TAVI & \multicolumn{2}{|c|}{$£ 30200(£ 27829, £ 32833)$} & $2.36(2.19,2.43)$ & \\
\hline MM & \multicolumn{2}{|c|}{$£ 5000$ (£3995, £6005) } & $0.80(0.61,1.02)$ & \\
\hline Difference & \multicolumn{2}{|c|}{$+£ 25200$} & +1.56 & 16200 \\
\hline Arm & \multicolumn{2}{|c|}{ Costs* } & OALYs & $\begin{array}{l}\text { ICER } \\
\text { ( }(\text { /OALY gained) }\end{array}$ \\
\hline \multicolumn{5}{|c|}{ Analysis 1: Pooled parameter values and PARTNER-B survival data } \\
\hline TAVI & \multicolumn{2}{|c|}{$£ 30600(£ 27758, £ 32946)$} & $2.34(2.19,2.43)$ & \\
\hline MM & \multicolumn{2}{|c|}{$£ 5000(£ 4042, £ 6101)$} & $0.80(0.62,1.02)$ & \\
\hline Difference & \multicolumn{2}{|c|}{$+£ 25600$} & +1.54 & $£ 16600$ \\
\hline Source & & 24 month survival & Derived multiplier $\neq$ & $\begin{array}{l}\text { ICER } \\
\text { ( }(\text { /OALY gained) }\end{array}$ \\
\hline \multicolumn{5}{|c|}{ Analysis 2: Impact of using alternative data sources for medical management mortality } \\
\hline \multicolumn{5}{|c|}{ Leon et $a^{14} \quad 50.3 \% \quad N A$} \\
\hline \multicolumn{2}{|l|}{ Dewey et $a l^{8}$} & $81.0 \%$ & 0.63 & $£ 14300$ \\
\hline \multicolumn{2}{|l|}{ Bouma et $a^{25}$} & $44.1 \%$ & 1.14 & $£ 18500$ \\
\hline \multicolumn{2}{|c|}{ Varadarajan et $\left.a\right|^{26}$} & $56.7 \%$ & 0.89 & $£ 16100$ \\
\hline \multirow[b]{2}{*}{ Source } & \multirow{2}{*}{\multicolumn{2}{|c|}{ Baseline decrement }} & ICER ( $($ /0ALY gained) & \\
\hline & & & Immediate benefit & $\begin{array}{l}\text { Time-dependent } \\
\text { benefit }\end{array}$ \\
\hline \multicolumn{5}{|c|}{ Analysis 3: Impact of using alternative sources and assumptions for TAVI-related utility } \\
\hline SF-6D & \multicolumn{2}{|c|}{$0.107 \S$} & $£ 17700$ & $£ 18400$ \\
\hline EQ-5D & \multicolumn{2}{|c|}{0.1539} & $£ 16900$ & $£ 17600$ \\
\hline NYHA III & \multicolumn{2}{|c|}{$0.187^{* *}$} & $£ 16400$ & $£ 17000$ \\
\hline NYHA IV & \multicolumn{2}{|c|}{$0.357 \dagger \dagger$} & $£ 19500$ & $£ 14500$ \\
\hline PARTNER & \multicolumn{2}{|c|}{$0.201 \neq \ddagger$} & $£ 17500$ & $£ 16700$ \\
\hline
\end{tabular}

*Rounded to nearest $£ 100$.

†Probabilistic rather than deterministic outputs. Information corresponds to mean and $95 \%$ credibility intervals.

$\ddagger$ Represents the ratio of study specific and PARTNER 24-month survival estimates. Applied to the baseline probabilities in both treatment and comparator arm.

§Derived from information in Bach et $a l^{27}$ using mapping algorithm presented in Ara et al. ${ }^{28}$ TBased on information in COR 2006-02 study: Medtronic data on file.

** Severe aortic stenosis assumed to be same as NYHA III. Value taken from Fox et al. ${ }^{20}$ $\dagger+$ Severe aortic stenosis assumed to be same as NYHA IV. Value taken from Fox et al. ${ }^{20}$ $\ddagger \ddagger$ Published values used in combination with baseline NYHA mix to generate average decrement.

GW, general ward; ICER, incremental cost-effectiveness ratio; MM, medical management; NYHA, New York Heart Association; QALY, quality-adjusted life year; TAVI, transcatheter aortic valve implantation. the 10-year costs and benefits associated with each treatment option reported in analysis 1 in table 2 are generated. The results are in line with the base case analysis, indicating that the model is insensitive to changes in these parameters.

\section{Truncation of time horizon}

We explored the impact of using a truncated time horizon over which costs and benefits were extrapolated (figure $2 \mathrm{~B}$ ). At costeffectiveness thresholds of $£ 20000$ and $£ 30000$ per QALY gained, TAVI became cost-effective after approximately 6 and 4 years, respectively. Thus, 2 years of extrapolation beyond the limit of the trial is required before TAVI becomes cost-effective.

\section{Choice of baseline survival curve}

The results from this analysis are presented in analysis 2 in table 2. Regardless of the choice of multiplier, the resulting ICER is below $£ 20000$ per OALY gained. Thus the model is not sensitive to choice of patient group.

\section{Use of alternative approaches to modelling utility}

In addition to different sources for the utility decrement associated with severe aortic stenosis, the improvement in HRQoL following TAVI can either be immediate or time-dependent-that is, the benefits of treatment can either be assumed to occur immediately after the operation or gradually over a period of time. The results from different combinations of these different assumptions are presented in analysis 3 in table 2 and have only a modest impact on the ICER.

\section{Additional sensitivity analyses}

In addition, extensive deterministic one-way analyses undertaken by systematically altering individual parameters by $\pm 10 \%$ highlighted that the model was very sensitive to changes to the short-term treatment effect and the cost of the initial operation. The model was robust to changes to hospitalisation costs and adverse event rates (figure 2C). In particular, complete removal of BAV from the medical management arm (the most optimistic possible perspective) resulted in an increase in the ICER to $£ 18500$ per OALY gained.

\section{DISCUSSION}

To the best of our knowledge, the model presented in this paper is the first to assess the cost-effectiveness of TAVI in inoperable elderly patients with multiple comorbidities using a lifetime time horizon. The results from the PARTNER-B economic model were presented at the 2011 American College of Cardiology meeting, but these were derived using a trial-based approach and hence may not have captured all of the long-term costs and benefits of TAVI and medical management. ${ }^{29}$ In constructing this model we used data from a range of sources and performed extensive sensitivity analyses to explore the robustness of our model parameters. Our cost-effectiveness model did not differentiate between different TAVI platforms. Thus, while a number of key parameters were based on data from the PARTNER US trial (cohort B), ${ }^{14}$ other model parameters (eg, adverse event rates, baseline survival and utility parameter values) were informed by an extensive literature review.

In particular, where alternative choices were possible as to data sources or parameter values, we intentionally chose those which would most bias against TAVI in order to generate conservative results. We accept that, for several parameters (mean ICU stay and complication rates), estimates are higher than might be observed in routine UK clinical practice. 

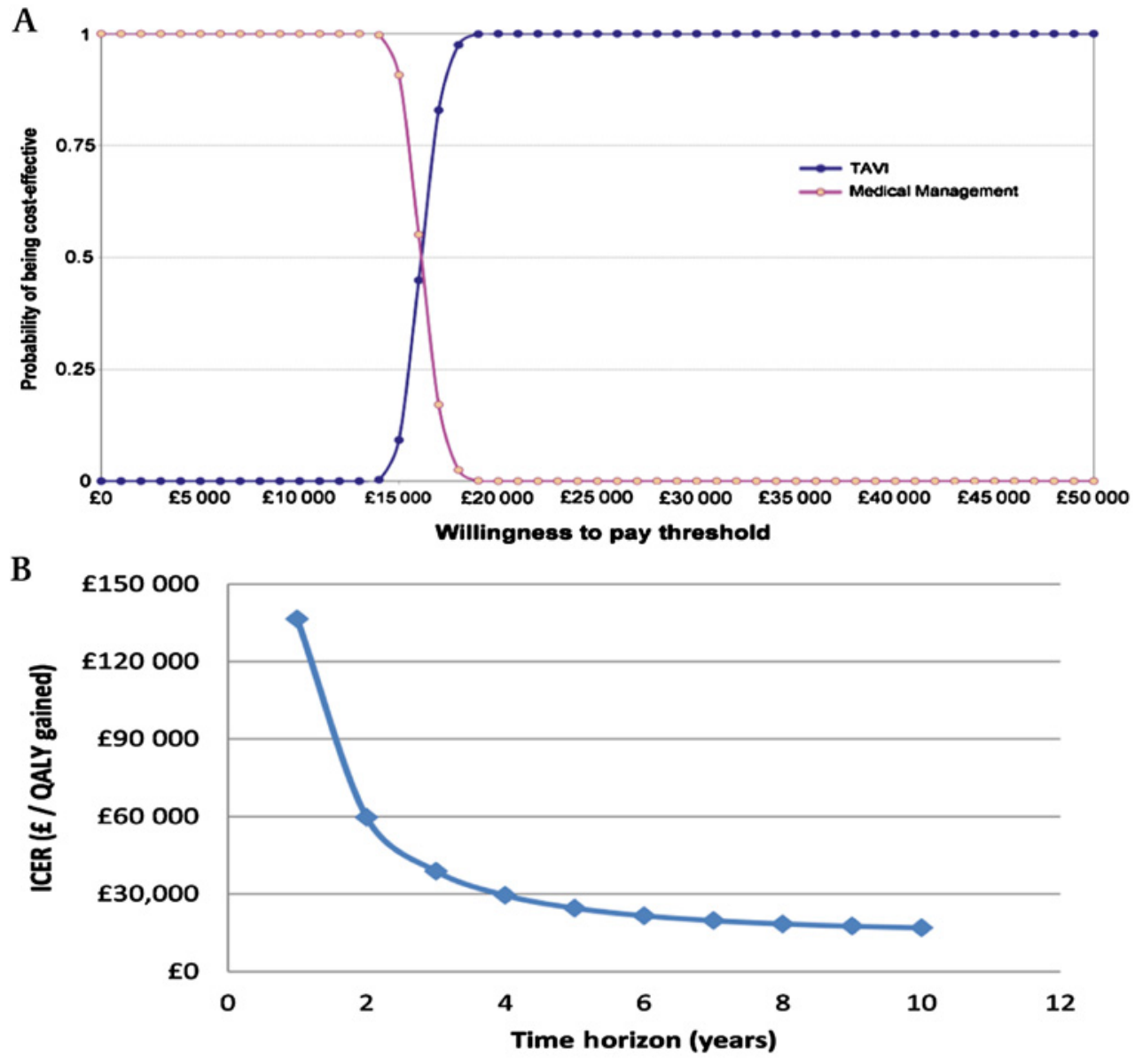

C

Tornado Diagram
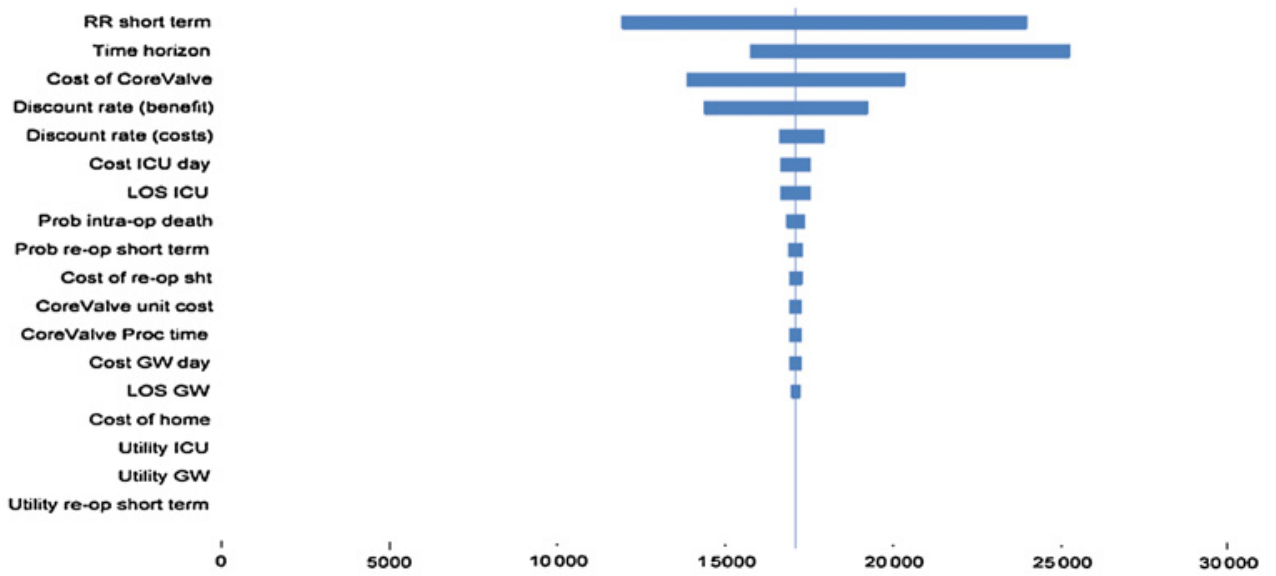

Figure 2 Graphical output from selected sensitivity analyses. (A) Cost-effectiveness acceptability curves showing the probability that each intervention is cost-effective at a range of cost-effectiveness thresholds. (B) Impact of alternative time horizons on cost-effectiveness. (C) Tornado plot showing results from deterministic one-way analyses. GW, general ward; ICER, incremental cost-effectiveness ratio; ICU, intensive care unit; LOS, length of stay; TAVI, transcatheter aortic valve implantation.

This model demonstrates that TAVI is highly likely to represent a cost-effective use of resources at cost-effectiveness thresholds typically used by the National Institute for Health and Clinical Excellence. The base case ICER was approximately $£ 16100$ per OALY gained. The robustness of the model was demonstrated after similar results were obtained using alternative parameter values from the TAVI literature. Of note is that the results were generated using a strict costing methodology whereby only costs directly incurred by the healthcare sector are considered relevant. Were a broader perspective to be used whereby additional costs were considered, any therapeutic benefit of TAVI would result in additional cost savings and hence a lowering of the ICER.

Analysis of the reported PARTNER data ${ }^{14}$ showed that the treatment effect associated with TAVI was not constant but increased over time. Moreover, patients receiving standard care experienced an extremely poor prognosis, with predicted 1 -year and 2 -year mortality rates of approximately $50 \%$ and $66 \%$, 
respectively. Information identified during the literature search in combination with parametric (Weibull) survival functions were used to generate alternative 2-year mortality values for patients receiving medical management. ${ }^{8} 2526$ Thus, the patient populations modelled in the sensitivity analysis surrounding choice of baseline survival curve are very heterogeneous. As such, these alternative data sources can be viewed as a proxy for different patient groups and hence will go some way to address the issue of off-label TAVI usage. Nonetheless, TAVI remained cost-effective regardless of which choice of group was made.

The results also show that whether the HRQoL benefits associated with TAVI are felt immediately or gradually over time had no major impact on the cost-effectiveness of TAVI compared with medical management. Further, the choice of baseline utility decrement used to include disease severity on the model also had no significant effect on the model results.

Similarly, imputing short-term adverse event data from the PARTNER US trial or from the broader literature into the model did not impact on the cost-effectiveness ratio. In all circumstances, TAVI remained a cost-effective intervention for the treatment of patients with severe aortic stenosis who are currently ineligible for surgery.

\section{Study limitations}

Despite the above-mentioned strengths, the key limitation to the analysis is the need to extrapolate reported data over a long time frame. The results from the sensitivity analyses showed that data from 24 months need to be extrapolated for at least 2 years to achieve cost-effectiveness. In particular, a small number of individuals contributed information presented in the Kaplan-Meier plot in the second year.

Long-term follow-up data are therefore required in order to overcome this. Given that TAVI has been shown to be clinically efficacious in the target patient group, there are obvious ethical issues in running future TAVI-related trials relating to randomising individuals to an apparently clinically inferior treatment option. Hence, such data may never be collected. Fortunately, the cost-effectiveness results derived from our exploratory analysis of the alternative data sources suggest that long-term extrapolation of trial data may not be required to demonstrate cost-effectiveness.

Similarly, the assumptions used to model device failure rates are not based on information from patients undergoing TAVI but from those receiving standard prosthetic valves and, as such, are an area where more accurate data would be required. However, 3-year TAVI failure data are available, with no failures reported over this time. ${ }^{12}$ Thus, it is highly likely that the implanted valves will outlive the people who receive them and that this limitation will not impact on the cost-effectiveness of TAVI.

Finally, the paper does not address the issue of the costeffectiveness of TAVI in patients who are currently eligible for SAVR. The rationale for this was that this patient group was likely to be different clinically from those who do not undergo SAVR, and the model may have different drivers of cost-effectiveness. The use of TAVI in this patient group will be the subject of a subsequent paper by this research group.

\section{CONCLUSIONS}

From a UK reimbursement perspective, in patients with severe aortic stenosis who are deemed ineligible for SAVR, it is highly likely that TAVI represents a cost-effective treatment option at cost-effectiveness thresholds typically used in decisions made by the National Institute for Health and Clinical Excellence.

Funding The project was funded by Medtronic International Trading Sàrl. However, no restriction was placed on either the design of the model or the presentation of results.

Correction notice This paper has been corrected since it was published online first. The percentage of intraoperative death in paragraph 4 of 'Description of model inputs', 'Estimating mortality', has been corrected from $0.8 \%$ to $1.1 \%$.

Competing interests MW, SM, JE and MS work for an international consultancy company and as such have received funding from numerous device and pharmaceutical companies to undertake studies similar to that presented in this paper. PB and RB are employees of Medtronic International Trading Sàrl. NP, NM and SP have received honoraria for the provision of expert advice throughout the project. MS, SP, NM and NP have undertaken consultancy for transcatheter aortic valve implantation (TAVI) manufacturers.

Contributors All authors contributed to the design, parameterisation and validation of the economic model. MW and SM constructed the economic model and prepared the manuscript. All authors provided significant comment on the content.

Provenance and peer review Not commissioned; externally peer reviewed.

\section{REFERENCES}

1. Carabello BA, Paulus WJ. Aortic stenosis. Lancet 2009;373:956-66.

2. Davies SW, Gershlick AH, Balcon R. Progression of valvar aortic stenosis: a long term retrospective study. Eur Heart J 1991;12:10-14.

3. Bonow R0, Carabello BA, Chatterjee K, et al. ACC/AHA 2006 practice guidelines for the management of patients with valvular heart disease: executive summary. $J \mathrm{Am}$ Coll Cardiol 2006;48:598-675

4. Turina M, Hess 0 , Sepulcri F, et al. Spontaneous course of aortic valve disease. Eur Heart J 1987:8:471-83.

5. Cribier A, Eltchaninoff $H$, Bash A. Percutaneous transcatheter implantation of an aortic valve prosthesis for calcific aortic stenosis: first human case description. Circulation 2002:106:3006.

6. Nishimura RA, Grantham JA, Connolly HM, et al. Low-output, low-gradient aortic stenosis in patients with depressed left ventricular systolic function: the clinical utility of the dobutamine challenge in the catheterization laboratory [see comment]. Circulation 2002;106:809-13.

7. Bleiziffer S, Ruge H, Mazzitelli D, et al. Results of percutaneous and transapical transcather aortic valve implantation performed by a surgical team. Eur J Cardiothorac Surg 2009;35:615-21.

8. Dewey TM, Brown DL, Das TS. High-risk patients referred for transcatheter aortic valve implantation: management and outcomes. Ann Thorac Surg 2008;86:1450-7.

9. CoreValve ReValving Experience. Aortic Valve Implantation by Subclavian Access Site. Presented at EuroPCR 2009; May 19-22, 2009. Barcelona, Spain.

10. Rodés-Cabau J, Dumont $E$, De LaRochellière $R$, et al. Feasibility and initial results of percutaneous aortic valve implantation including selection of the transfemoral or transapical approach in patients with severe aortic stenosis. Am J Cardiol 2008:102:1240-6.

11. Thomas M, Schymik G, Walther T, et al. Thirty-day results of the SAPIEN aortic Bioprosthesis European Outcome (SOURCE) Registry: a European registry of transcatheter aortic valve implantation using the Edwards SAPIEN valve. Circulation 2010;122:62-9.

12. Webb JG, Altwegg L, Boone RH, et al. Transcatheter aortic valve implantation. Impact on clinical and valve-related outcomes. Circulation 2009;119:3009-16.

13. Tamburino C, Capodanno D, Mule M, et al. Procedural success and 30-day clinical outcomes after percutaneous aortic valve replacement using current third-generation self-expanding CoreValve prothesis. J Invasive Cardiol 2009;21:93-8

14. Leon MB, Smith CR, Mack M, et al. Transcatheter aortic-valve implantation for aortic stenosis in patients who cannot undergo surgery. N Engl J Med 2010;363:1597-607.

15. Raikou M, McGuire A, Lurz $\mathrm{P}$, et al. An assessment of the cost of percutaneous pulmonary valve implantation (PPVI) versus surgical pulmonary valve replacement (PVR) in patients with right ventricular outflow tract dysfunction. J Med Econ 2011:14:47-52.

16. National Health Service. NHS Reference Costs. http://www.dh.gov.uk/en/ Publicationsandstatistics/Publications/PublicationsPolicyAndGuidance/DH 062884

17. Curtis L. Unit Costs of Health and Social Care. http://www.pssru.ac.uk/pdf/uc/ uc2008/uc2008.pdf

18. British National Formulary. BNF 56. http://www.bnf.org/bnf

19. Kind P, Hardman G, Macran S. UK Population Norms for EQ-5D. [172]. 1999 CHE Discussion Papers. http://www.york.ac.uk/media/che/documents/papers/ discussionpapers/CHE\%20Discussion\%20Paper\%20172.pdf

20. Fox M, Mealing S, Anderson R, et al. The clinical effectiveness and costeffectiveness of cardiac resynchronisation therapy (biventricular pacing) for heart failure: systematic review and economic model. Health Technol Assess 2007;11: iii-iv, ix-248. 
21. Edwards SJ, Campbell HE, Plumb JM. Cost-utility analysis comparing meropenem with imipenem plus cilastatin in the treatment of severe infections in intensive care. Eur J Health Econ 2006; 7:72-8.

22. Buellesfeld L, et al. 12 Months Safety and Performance Results of Transcatheter Aortic valve Implantation Using the $18 \mathrm{~F}$ CoreValve Revalving Prosthesis. Barcelona, Spain: EuroPCR, 2009

23. Puvimanasinghe JP, Steyerberg EW, Takkenberg JJ, et al. Prognosis after aortic valve replacement with a bioprosthesis: predictions based on meta-analysis and microsimulation. Circulation 2001:103:1535-41.

24. Drummond M, Sculpher M, Torrance G, et al. Methods for the Economic Evaluation of Health Care Programmes. Oxford, Oxford University Press. 2005.

25. Bouma BJ, van Den Brink RB, van der Meulen JH, et al. To operate or not on elderly patients with aortic stenosis: the decision and its consequences. Heart 1999;82:143-8.
26. Varadarajan P, Kapoor N, Bansal RC. Clinical profile and natural history of 453 nonsurgically managed patients with severe aortic stenosis. Ann Thorac Surg 2006;82:2111-15.

27. Bach DS, Cimino N, Deeb GM. Unoperated patients with severe aortic stenosis. $J$ Am Coll Cardiol 2007; 50:2018-19.

28. Ara R, Brazier J. Deriving an algorithm to convert the eight mean SF-36 dimension scores into a mean EQ-5D preference-based score from published studies (where patient level data are not available). Value Health 2008; 11:1131-43.

29. Reynolds MR, et al. Lifetime Cost Effectiveness of Transcatheter Aortic Valve Replacement Compared with Standard Care Among Inoperable Patients with Severe Aortic Stenosis: Results from the PARTNER Trial (Cohort B). American College of Cardiology Annual Meeting. April 2011.

\section{Images in cardiology}

\section{The use of positron emission tomography in the diagnosis of pacemaker related infection}

A 59-year-old man underwent dual chamber permanent pacemaker (DDD PPM) implantation for complete heart block following emergency mechanical mitral valve replacement and coronary artery bypass grafting.

At routine pacemaker check 5 months after the implant, a painless moderate sized swelling persisted over the box. The
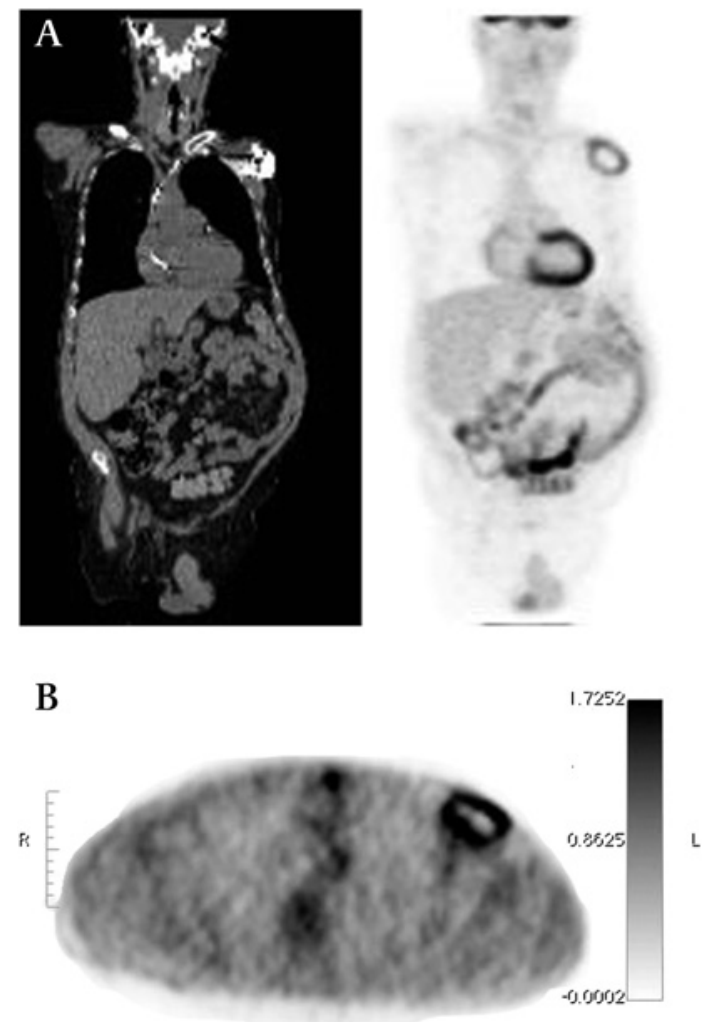

Figure 1 Fluorodeoxyglucose positron emission tomography images demonstrating increased uptake associated with the left pectoral permanent pacemaker implant site. patient described several episodes of rigours and sweating in the preceding month. The patient was otherwise systemically well and without fever. Blood tests demonstrated a normal white blood cell count $\left(9.9 \times 10^{9}\right)$ and erythrocyte sedimentation rate $(13 \mathrm{~mm} / \mathrm{h})$ and a mildly raised $\mathrm{C}$ reactive protein $(11 \mathrm{mg} / \mathrm{l})$. Chest radiograph was unremarkable. Blood cultures were negative. A transoesophageal echocardiogram did not show evidence of intracardiac vegetation.

$\mathrm{He}$ underwent a fluorodeoxyglucose positron emission tomography (FDG-PET) scan which demonstrated significantly increased uptake associated with the PPM implant site and no uptake in the region of the mitral valve replacement or pacing lead (figure 1)

We successfully extracted the infected PPM system observing frank pus on opening the wound. Wound pus, debrided tissue and pacing lead culture demonstrated coagulase negative Staphylococcus epidermidis and Candida albicans. The patient received a 2 -week course of intravenous vancomycin and oral fluconazole followed by uneventful DDD PPM reimplantation on the contralateral side.Discussion

FDG-PET imaging may be useful in the diagnosis of suspected PPM infection in which there is a fever of unknown origin and normal baseline investigations including transoesophageal echocardiogram. ${ }^{1}$ Repeat pacing interventions are not without risk particularly of bleeding, haematoma and introducing infection. In this case, FDG-PET imaging correctly identified the underlying PPM-related infection that ultimately aided in successful treatment.

\section{Paresh A Mehta, Zia Zuberi, C Aldo Rinaldi}

Department of Cardiology, St Thomas' Hospital, London, UK

Correspondence to Dr Paresh A Mehta, Department of Cardiology, St Thomas' Hospital, Westminster Bridge Road, London SE1 7EH, UK; p.mehta@imperial.ac.uk

Contributors All authors contributed equally to the writing of this case.

Provenance and peer review Not commissioned; internally peer reviewed.

Published Online First 19 November 2011

Heart 2012;98:376. doi:10.1136/heartjn-2011-301138

\section{REFERENCE}

1. Ploux S, Riviere A, Amraoui S, et al. Positron emission tomography in patients with suspected pacing system infections may play a critical role in difficult cases. Heart Rhythm 2011;8:1478-81. 